\title{
A Novel Primary Healthcare Physician Associate Internship: Results from a Service Evaluation
}

Elizabeth Cottrell ( $\square$ e.cottrell@keele.ac.uk)

Keele University https://orcid.org/0000-0002-5757-1854

\section{Victoria Silverwood}

Keele University School of Medicine

\section{Lucy Minshull}

North Staffordshire GP Federation

John J Edwards

Keele University School of Medicine

Alex Strivens-Joyce

Montgomery Medical Practice

Matt Aiello

Health Education England London

\section{Sarah Lawton}

Keele University School of Medicine

\section{Sharon Turner}

North Staffordshire GP Federation

\section{Research article}

Keywords: Physician associate, internship, primary care, evaluation, integration, implementation

DOI: https://doi.org/10.21203/rs.3.rs-70742/v1

License: (9) This work is licensed under a Creative Commons Attribution 4.0 International License.

Read Full License 


\section{Abstract}

Background: Physician associates (PA) are dependent healthcare professionals with two-years postgraduate generalist medical education. PAs can work in primary care immediately after graduation. However, PAs are novel to, and still relatively uncommon within, UK primary care. Recognising the challenges presented by UK primary care, including the undifferentiated, complex sociomedical and nonprotocolised presentations, a novel one-year internship scheme was established to support PAs to work in primary care and to support PA retention in primary care and within the geographical region. This evaluation sought to gain feedback to guide further roll-out and development of the internship and to establish its success in supporting PAs into primary care.

Methods: The Staffordshire PA Internship (SPAI) scheme was introduced in Staffordshire in 2017. PAs worked concurrently in primary and secondary care posts for one year, with protected weekly primary care focussed education sessions. Posts were subsidised and PAs were centrally employed. Evaluation data pertaining to the first two cohorts were sought from the PA interns, supervising GPs and practice managers.

Results: By evaluation end, eight of the ten PAs had completed the internship. Practices valued the subsidised, central employment model of the internship as it provided a risk-limited opportunity to explore the PA role, which iteratively developed to establish weekly timetables that best suited PAs and practices. While the one-year PA programme had seen PA interns develop significantly from the start, many practices and PAs felt that a two year programme may have been preferable. There were mixed views about whether a split post or sequential posts may be preferable, however, PAs did not have experience of the latter.

Conclusions: This test-of-concept SPAI was successful in retaining PAs in primary care in the geographical area of the internship. However, it highlighted the need for PA interns to have intensive supervision, particularly in the early weeks, and a dedicated educational programme, which was only possible due to the internship arrangements. The optimal internship design remains unknown, however, sole primary care internship posts may accelerate upskilling of PA interns for the challenges of primary care and may better support their integration within practices.

\section{Introduction}

Physician Associates (PAs) are dependent healthcare professionals who have a generalist medical education. Having undertaken a biomedical or healthcare related undergraduate degree, PA clinical training is completed over two years. Successful completion of a national exam enables PAs to join the Royal College of Physicians' (RCP) Physician Associate Managed Voluntary Register (PAMVR)[1]. Until 2019, PA clinical training was only required to include four weeks' experience within primary care. Once PAs have completed PA school they can enter autonomous primary care roles, under the supervision of a general practitioner (GP). 
The PA role is often not familiar to patients and healthcare professionals in UK primary care as PAs are novel to, and uncommon within this setting[2, 3]. In 2018, fewer than 200 PAs were working in UK primary care[2,3]. However, policy changes have driven the training of 1,000 new PAs to support primary care in order to deliver the NHS Long Term Plan[5] through Primary Care Networks (PCNs) [6]. Current UK primary care PA workforce plans and understanding of the role are predominantly based on USA data. These data may have limited generalisability to the UK, as within the USA, PAs are both more established and have wider rights, such as prescribing and ordering ionising radiation. UK PAs are not yet able to undertake these activities until regulation arrangements are in place. In the meantime supervising GPs have to complete these tasks for PAs. No central, dedicated funding or support had been provided at the initiation of these policy driven changes for new PAs leaving their course. Further, the continuing professional development needs of PAs working in primary care had not been explicitly laid out or standardised at their introduction to primary care[7].

Recognising the forthcoming policy-driven workforce changes, particular primary care workforce issues in the local health economy and the potential benefit of PAs working in primary care, workforce leads in Staffordshire prioritised developing this workforce. They predicted that for successful integration and implementation of PAs into primary care, and to encourage local primary care retention, additional, dedicated, primary care post-graduate support would be required. The Staffordshire PA internship (SPAI) scheme was thus born. The overall aim of internship evaluation was to establish how the PA internship supported the integration and implementation of PAs into primary care. This evaluation sought to gain feedback to guide further roll-out and development of this novel scheme and to establish the internship's success in supporting PAs into primary care.

\section{Method}

\section{The Staffordshire Physician Associate Internship (SPAl)}

In October 2017, the first SPAl cohort commenced. At this time, several practices were becoming unsustainable and facing closure. The SPAI was delivered by a Clinical Lead (GP), Project Manager, PA Ambassador and experienced GP tutors. Initially the SPAI was designed as a single-year pilot split-post longitudinal scheme, such that PAs worked for one year in a single practice and a secondary care post (see Fig. 1). However, in the second cohort, two PA interns were working in full-time primary care roles.

With no explicit or standardised approaches to primary care PA continuing professional development (10), strategies to support new PAs into primary care were developed by the SPAI team based on expert opinion, drawing on expertise in training other professional roles. The education curriculum, delivered over the year in half-day protected teaching sessions, was designed to address all key primary care clinical topics.

Workforce leads within the North Staffordshire GP Federation (NSGPF) worked in partnership with five local NHS trusts to develop the SPAl, with a single Trust as a central host employer. This central 
employment model, meant that practices were not required to employ the PA interns. Recruitment processes and pre-employment checks were undertaken by the central employer and the NSGPF. PAs who were selected for the internship were matched by the SPAI team matched to practices, based on knowledge of where the PA Intern lived and the practice location and mutual interests. Initially, the internship cohort start date was designed to dovetail with the PA's national exams to reduce the time spent unemployed between qualification and commencement of the internship.

The SPAI had a mixed funding model. The five NHS Trusts provided the salary for the secondary care posts, host primary care practices paid half the costs for salary, on-costs and indemnity (required at the time), with NHS England subsidising the remaining primary care costs. Health Education England funded the learning programme, GP tutor, a practice supervision fee, materials and separate funding for this evaluation.

\section{Data collection}

Lau et al.'s conceptual framework for implementing interventions in primary care[8] was used to develop the data collection tools. Evaluation data were sought from the first two SPAl cohorts (commencement October 2017 and March 2018) at three time points by the same evaluator (EC; start, mid-point and end of each cohort). PA intern data were obtained through both questionnaire surveys and discussion groups. Practice data were collected through in-practice discussions with the practice manager and/or supervising GP for the intern. Reasons for practices not engaging with the internship were sought using a questionnaire survey (September 2018) from the 121 practices in the area who could have, but did not, not host an intern.

\section{Data Analysis}

Results from both cohorts were combined. Discussion groups were audio recorded and transcribed. Data analysis was guided by Lau's conceptual framework such that: the 'external context' related to data describing issues relating to the local and national health economies; the 'organisation' data were those related to the host primary care practice; the 'professional' domain contained data relating to the PA Intern; and the 'intervention' data were those referring to the SPAI. Discussion data that mapped to quantitative survey data were coded as such and amalgamated into a quantitative evaluation data sets. Closed and multiple-choice survey responses were coded in IPM SPSS and descriptively analysed. Open responses from discussions and survey data were thematically analysed using our evaluation framework. Concepts raised from open questions were independently identified and coded by two evaluators (EC and VS). While the evaluation framework remained set throughout the analysis, subthemes and concepts arising from these were developed iteratively. Concepts where then mapped together within the framework, linking supporting concepts and identifying areas of dissonance.

\section{Results}

Overall, data were collected from all ten PAs at baseline (seven gave data at every time-point), eight of the nine practices at baseline (six gave data at every time-points), and 28 of the 121 practices who did not 


\section{Internship Design}

The year-long, split-post longitudinal design was debated by PA interns and practices. There was no clear consensus about how the internship should be structured moving forwards. See Box 1 for illustrative quotes.

\section{Internship duration}

Practices and PA interns reported mixed views about the most appropriate duration of the Internship. The time-limited approach to the internship was felt to be beneficial to reduce the perceived risk of engaging with the internship; however many respondents felt that the internship should be longer. It was suggested that a two-year internship might allow PA interns to develop more established skills in managing the more complex, multi-morbid patients (for example, those in nursing homes) and to develop a broader expertise as certain conditions are not seen at regular frequency in a generalist setting. By the mid-point of the Internship, some PA interns were working more autonomously, had visibly growing confidence (according to host practices) and were requiring less supervision. However, even at the end of the Internship, practices who reported their PA interns as having progressed well, still reported unrealised development ideas/requirements for the PA interns.

\section{Split primary and secondary care posts}

The split-post model was derived out of necessity to accommodate the available funding. PA interns liked the variety and the wider expertise that the split post brought, for example, the experiential knowledge of one post, supporting the work in the other. However PA interns reported difficulties integrating themselves simultaneously into two new roles. Initially, the split-post nature of the model highlighted to PA interns the difficulties of working in the undifferentiated primary care context. Their secondary care posts were felt to be more protocol-driven with easier access to immediate support for prescriptions and queries. However, PA interns shifted their preferences over time, recognising the benefits of autonomous working and the varied work within primary care.

\section{Concurrent longitudinal design}

The one year split post, as opposed to two six month posts, was designed to support trust and long-term working relationships to be established between the PA intern and their supervisors. Indeed, this was felt to be beneficial in allowing GPs and PAs interns to build trust in and an understanding of each other over time. However, this design introduced the cost of slower integration and establishment of roles and mutual trust. Practices valued the opportunity to train the PA intern over the year to shape them into the type of professional they wanted at the practice, with a view to employing them at the end of the year internship. 
The split-post longitudinal design, which resulted in the PA interns only being in the practice part time, did create some difficulties for practices. Being absent from practice two days a week resulted in missed opportunities to participate in fixed activities, such as locality based protected learning time. Practices who did not have full time PA interns were keen to do so as they anticipated that this would accelerated the development of, and trust in, the PA intern and would support consistent staffing levels and care navigation approaches. PA interns also recognised it would enable continuity in results handling and would mitigate against the burden of having to establish oneself in two jobs at the same time. The prospect of having a PA full time, did not necessarily mean the practice thought the duration could remain at a year, one GP called for a longer, full-time internship. Not all practices wanted a full-time PA, due to room capacity.

Models involving sequential 'blocks' of placements, of varying duration, were suggested, such as those undertaken by junior doctors. Often it was the PA interns who wanted this approach. However, feedback from practices suggested that shorter 'blocks' may hinder development of a meaningful, trusting relationship between PA interns and supervisors, and practices implied they may have reduced motivation to invest large amounts of time and effort in developing the PA intern within short blocks, thus potentially stunting the PA's development.

\section{Internship start timing}

Between cohort 1 and cohort 2 the timing of the internship commencement altered in response to the relatively high fail rates from the PA exams. In cohort 1, practices had prepared for a PA intern (including setting up indemnity cover) but the intern failed exams/declined internship at the last minute, this created problems for practices. The start date for the second cohort date was pushed back, to enable set up post results; no further feedback about the timing of the start date was then received.

\section{Central and subsidised employment model}

The central employment provided a safety net in the event of supervisor-intern problems and it mitigated against financial risk posed by sickness or maternity leave. Practices also suggested that the lack of familiarity of PAs within primary care would have been a barrier to recruitment without the central employment model. The main difficulty practices reported with this central employment model was uncertainty about what would and should happen in the event of maternity (e.g. will the PA intern return to the practice?) and sick leave (e.g. should the practice hold a return to work interview?).

The lack of clearly defined roles for PAs in general, and particularly new PAs such as the interns, in primary presented practices with challenges as they were often unfamiliar with the role. The subsided internship model allowed for practices and PA interns to test out different activities in a relatively inexpensive and low risk way. This had great benefits as many PA interns were doing roles at the end of the internship that they, and their practices, had not envisaged at the outset and practices learnt which PA roles work and do not work in their own organisation. 


\section{Internship duration}

“...[the PA] is being expected to be expert after one year and I don't think any of us would have said we were expert after one year" (GP)

"I think if the internship was longer and we had more teaching around chronic disease management... and had to manage patients as complex patients, I think it would have been more beneficial...the internship's ended and we're just left to get on with it." (PA intern)

\section{Split-post}

"...so if say if I had a patient come in with....an abscess and I needed to refer them to the surgical assessment unit at the hospital, l'd understand the process that patient has to go through so who to refer to, what they have to go through so then I can give that patient a bit more insight in what's actually gonna happen...so I think it helps having hospital and GP experience." (PA intern)

"...it's a good idea with them being split...See initially, I think [the PA] does enjoy the secondary care aspect of the variety but it's very different to primary care isn't it so here, maybe [the PA] feels more trustworthy...[the PA]'s consulting a patient on an one to one basis...taking that history and then the GP partner's are trusting [the PA] to diagnose and...leave the premises..." (PM)

\section{Concurrent longitudinal post}

"I'm in two minds over the split days thing...it's nice to be able to have a break away from the other speciality but...sometimes the staff will forget and I'll get sent urgent tasks...If I'd have been in all week, I could have seen that and...the staff might get to know you a bit better as well..." (PA intern)

"I would make it twice as long I think and I would probably, instead of doing a 50/50, I'd make it a hundred percent general practice which is what we ended up with" (GP)

\section{Central and subsidised employment model}

"We just never would have [employed a PA without the internship] to be honest I don't think because the nice thing is the recruitment's done for us, so someone else is assessing who's a good candidate and who isn't and we did the recruitment for the pharmacist role but it was invaluable having a really experienced pharmacist from the CCG sat in on all the interviews 'cause they were able to help us work through the CVs, work out what was good, what wasn't good, what we wanted so that is quite a big burden and initial hurdle to overcome so the fact that that was all done for us is brilliant...So that was really good. I think if we were doing it all on our own, yes I just don't think we would have had the confidence to do it actually" (GP)

Box 1 Feedback on internship design

\section{Internship educational programme}

The weekly, primary care focussed, educational programme provided PA Interns with tailored training. A case-based approach was adopted to prompt learning from each others' experiences. PAs valued adapting previous learning to the primary care setting, where equipment, support and access to resources are different to the hospital setting. PA interns used the education programme to gain peer-to-peer support. PA interns preferred applied teaching styles, outlining how to operationalise guidelines in primary care rather than just what the guidelines say and they wanted information on prescribing; for example they cited a good session as one on eczema in which they were told how much emollient a patient should use and should be prescribed each month. 
Practices also valued the educational programme (see Box Two) because it provided reassurance that the PAs were being supported in a role-appropriate way; something they were less certain about due to their lack of familiarity with PAs. Practices found in-practice supervision to be resource intensive, thus, without the educational programme, they would not have been able to provide the same level of training. Indeed, providing post-Internship, ongoing education and continuing professional development for the PAs was something that concerned some practices.

"It's really nice to know that [the PA is] getting education outside the practice. I think that...the Wednesday afternoon thing is really helpful...[the PA] gets peer support which I think is really, really valuable,...gets to sense check how other people are doing which is crucial, [the PA will] bring back ideas about what works elsewhere which is really helpful so all of that peer support is invaluable" (GP)

Box 2 Illustrative quotes of the views of the internship educational programme

\section{Delivery of internship in practice}

\section{PA-intern patient boundaries}

Without a clearly defined role for PAs in primary care, practices adopted different strategies to outline the boundaries they felt were appropriate for them and their intern. These boundaries were sometimes guided by the PA intern and evolved throughout the internship. For example, a PA intern who was not seeing children at the beginning was able to see well children (for example, a child with eczema) by the midpoint. Not all PA interns set boundaries, and some practices reflected that the PA interns had fewer boundaries than their Nurse Practitioners.

\section{Use of red-amber-green (RAG) ratings to aid care navigation}

Some PA intern boundaries were formalised through the use of Red Amber Green (RAG) ratings, to highlight what the PA intern would see, would not see and would see under certain circumstances. This supported care-navigation purposes, however some PA interns and practices raised concerns about the impact of RAG ratings on maintaining holistic and generalist skills, necessary for revalidation. A pragmatic solution, suggested by a host GP, may be to define a RAG rating system according to competencies at internship start, and to focus tailored continuing professional development on items in the Amber and Red categories.

The alternative approach to using formalised RAG ratings is to see an undifferentiated workload and seek help once at least the initial assessment was done. This approach seemed to work best if GP supervisors were readily available (e.g. with appointment slots blocked for this purpose) to ensure that PAs and patients did not get frustrated and the GP was not (perceived to be) put under pressure by being made to run late. PA interns reported that after seeking help they would document the discussion to demonstrate the line of responsibility and that advice had been sought.

\section{Protocolised activities}


Practices reported PA interns undertaking protocol-driven activities, such as management of common, simple conditions (for example, simple urinary tract infections) and incoming results management. PAs liked protocol-driven approaches and perceived this to be more common in secondary care placements. PA interns highlighted that through increased use of protocols in primary care more autonomous working could be promoted. The commonly non-protocol-driven approach to GP management of patients, created confusion and uncertainty among PAs who felt they may not always know what to do next time. Further, PAs were not happy to deviate from guidelines.

In general, PA interns were only managing incoming results in practices in which other non-GP clinicians (e.g. Nurse Practitioners) were doing the same and pre-existing protocols to support this activity were already in place. Protocols for results handling often had clear parameters to determine normality and any results falling out-with these were sent to, or discussed with, a GP.

\section{Supervision approaches}

Host practices recognised the importance in investing time in the PA interns early in their internship however practices structured PA intern supervision in different ways. Some practices blocked out time whilst others provided support on a more flexible basis. It was acknowledged that for some practices providing support for the PA created additional workload and some practices had multiple dependent practitioners requiring GP support at the same time. It was acknowledge that training practices appeared to be better able to successfully integrate a new professional into the practice team as well as being more familiar with the inherent 'risks' of supporting dependent practitioners.

Practices that had invested a lot of time in the early months of the Internship in supervising their PA interns, seemed more likely to be satisfied with the role the PA intern was fulfilling by the end of the Internship. As time went on, face-to-face reviews of patients seen by PA interns did reduce and practices developed a variety of different ways of supervising and supporting the PA interns.

\section{Providing adequate time for induction to the entire practice}

Host practices recognised the importance in investing time in the PA interns early in their internship. Through sitting in and working with various different members of the team, a mutual understanding of the different roles emerged and team integration was supported. Practices recognised the importance of involving the PA interns in both clinical activities but also the administrative and business activities within the practice, to provide exposure to the wider context of primary care and the professional disciplines within it.

\section{Social interaction}

The most commonly mentioned factor directly aiding the integration of PA interns into the primary care team was being liked at a personal level. Social interactions appeared to critical for promoting primary care team integration. Practices often cited activities such as encouraging staff to have lunch in 
communal room and hosting social events as a strategy that aided all new practice team members to integrate with all staff types; PA interns were no exception.

\section{Training practices}

Training practices saw that they had transferrable skills and an existing culture that supported integration of new and inexperienced professionals to the team and an acceptance among patients that there was a turnover of clinicians providing care. Further, training practices were more familiar with the inherent 'risks' of supporting dependent practitioners.

\section{Extended appointment times}

The SPAl team provided guidance about appropriate appointment lengths over time, however, appointment length was often predominantly led by the PA interns' preferences and activities. Overall, PA interns were over-optimistic at internship start about their progress to reduce their appointment times. At baseline, eight of the ten interns had 30 minute appointments, with the remaining two having 20 minute appointments. While six believed that over the first three months they would progress to 15 minute appointments, by the end point, only two of the seven PAs within the internship were undertaking 15 minute appointments, four were on 20 minute appointments and one was still using 30 minute appointments. Factors cited as contributing ongoing use of longer appointment times, included the need for PA interns to seek input for prescriptions from GPs (which took time) and because undifferentiated patients, being managed in non-protocol-driven ways, was a particular and (possibly unexpected) challenge for the PA interns.

\section{Sources of support used by practices}

Nationally available information describing PAs provided information on the potential roles for PAs in general practice, but did not provide support for practices to integrate PAs new to primary care. Although these documents were referred to by the practices, they were felt be insufficient to support practices to implement PA interns, particularly PAs who were inexperienced in primary care. PAs and host practices commonly lacked clarity about what PAs are allowed to do (e.g. flu jabs), permissible ways for demonstration of competence (e.g. minor surgery) and medicolegal implications of activities (e.g. undertaking home visits). Host practices requested more formal guidance to assist with development, care navigation and to reassure that their activities were aligned with other practices. Practices wanted explicit, centrally produced, agreed and operationalisable guidelines.

The SPAI team became a heavily relied on resource to bridge this information gap. They responded to questions and requests for further nuanced information, provided support to practices, and solutionsought with practices when they perceived they were having problems. Issues identified during this evaluation led to the SPAI team activities increasing and a GP tutor role was developed to provide additional in-practice support to practices and PA interns.

\section{Satisfaction with the internship}


By the mid-point of the year, most PA interns and practices felt the internship was working well for them. This view continued among all practices who hosted a PA intern to internship completion. Benefits of the internship were identified by PA interns and practices whether or not the PA was going to remain in primary care or the practice was going to retain their/another PA intern graduate in practice after the internship. PAs felt that the internship was a supported way to better understand the primary care context.

\section{Internship value for money}

By the end of the internship practices generally felt that the scheme offered good value for money (see Box 3). However, initial value for money estimates, which focussed on cost-per-contact time were neutral, or when taking into account supervision, negative to practices. Despite recognising the initial lack of value for money, practices saw the investment opportunity; exploring the PA role in a low-risk way with potential to gain additional capacity over time.

Generally, the PA interns were described as more flexible than nursing staff but less flexible than GPs. This 'hybrid' role brought significant (potential) advantages to practices as PAs were perceived to be able to 'plug' the gap in service provision left by a wide range of clinical staff (permanently or while on leave). A value was seen in just being given opportunity to explore PA roles. Despite a growing value being seen in the PA interns over the internship year, practices could not get away from the inequity in central funding approaches for, the less experienced, PAs compared to GP specialty trainees.

"...they were about a third of a cost of a GP but they spend three times the length of time with the patient...so if you ask me whether, for the same cost, I would want...a GP...they have greater flexibility in their role" (GP)

"...we're in it for a longer gain and I think for the money we have to pay [the PA intern], we're exploring with no great long commitment a way of how do we teach PAs and what's their potential role so the value is that..." (GP)

Box 3 Feedback about perceived value for money of the internship

\section{Fit with local and national context}

\section{Effectiveness of the SPAl in retaining PAs in Staffordshire}

The SPAl appeared to be successful at attracting PAs to, and retaining them in the county. At the start of the Internship, of the ten responding PA interns, only four (40\%) felt it had been likely or very likely that they would have chosen to work in the area, two were not sure and the remaining four felt it to be (very) unlikely. By the end of the evaluation period, six of the eight interns who had completed their internship were staying in the county.

\section{Effectiveness of the SPAl in retaining PAs in primary care}

Retention of PAs in primary care is reliant on both practices and PA interns valuing and having interest in each other. At baseline PA interns reported feeling more autonomous in primary care. This autonomy 
(sometimes referred to as 'independent' within the feedback), could be perceived as a threat, or daunting, if the PA interns were or felt inadequately supported. However, autonomy and the ability to own consultations, without relying on a team of people, were reported as benefits of primary care by some PA interns (see Box 4). The importance of matching the PA interests to the practice needs was starting to emerge as practices learnt what the PA intern was capable of. By the end of the evaluation period, seven out of the eight PA interns who had left or completed the internship were (going to be) working in primary care. This compares positively with the baseline data which showed that only half of the 10 interns wanted to work solely in primary care with three thinking about a combined primary and secondary care role and two not being certain what they wanted. The one PA intern who reported that they were going to be taking on a secondary care post, reported that primary care remained a future option

"I think you are more involved as a clinician in primary care rather than secondary...[where I was]... finding myself like a clerk, like doing errand boy doing probably three or four hours discharge letters on what consultant say but here in primary care you're more independent. (PA intern)

"I think coming from [the hospital] environment where everybody works together and you've got as much time as you want to - this is your clinic, this is your times, see how you go and them are your patients. I think that was a bit of a ...A shock that it's more independent in general practice" (PM)

Box 4 Feedback about the effectiveness of retaining PAs in primary care

\section{Reasons for non-engagement with the internship}

The three top reasons for practices not engaging with the SPAI were: lack of space within the building for another staff member, reservations in the practice team about the PA and a perception that it did not represent good value for money. Additional reasons for not hosting a PA intern included, it not being the right time for the practice which had just expanded, already an active training practice, do not have a vacancy for a role like this, lack of clarity about what a PA will add and their role and fears about increasing the workload and issues relating to liability, indemnity and governance. Lack of inability to prescribe were cited among practice team member reservations about PAs, particularly if teams already included prescribing Advanced Nurse Practitioners and Pharmacists.

\section{Discussion}

\section{Summary}

The subsidised, time-limited, central employment model adopted for this PA internship allowed practices to 'test out' a PA in a low risk way and allowed PA interns to develop their experience of, and interest in, primary care. Although small numbers within the two cohorts, the SPAI appears to be effective at attracting and retaining PAs within primary care in the locality in which it is hosted. Some practices used the internship to shape the PA into the roles they wanted with a view to employing them at the end of the year. In this way the longitudinal post design, may have promoted retention, however this cannot be confidently concluded. There were mixed views about the optimal design of the internship, however, there was overall agreement that this should be lengthened to two years to equip PAs to develop the breadth of 
knowledge and experience required for the undifferentiated, complex and infrequent presentations. Tailored primary care PA education was seen as crucial; learning needs should be identified early on in a PA internship to allow for appropriate support and supervision to be provided.

Strengths and limitations

The strength of this service evaluation is that is describes a novel internship scheme for new PAs entering primary care. The learning from this has already started to shape future cohorts of the internship and lessons learned can inform the introduction of new PAs in to primary care networks. Further, many of the lessons learned are likely to be applicable to other novel roles within primary care. This evaluation builds on previous empirical work to develop an understanding of the UK context which is crucial at this time of significant policy driven change. All interns provided data within at least one time point, all but one practice provided data also, thus providing the widest possible range of views. Limitations of this evaluation are that participants were not required to complete every element of data collection and the pragmatic design, adopted to maximise engagement, meant that exact resource (time and financial) use could not be evaluated.

Comparison with existing literature

The perception of flexibility of PAs compared to nurse practitioners, for example, has previously been described by empirical literature[9]. While it seemed likely that by not setting boundaries the need for high levels of supervision may be higher, no evidence has been identified to demonstrate this. It is recognised that primary care focussed information for PAs and about employing PAs has improved over the course of the Internship (e.g. a dedicated website[10]) however, a more explicit central agreement and strategy developed with key stakeholders to ensure consistency across disciplines and to ensure priorities are aligned, is yet to be published. Ideal internship design, for example, split blocks (such as that in LIFT I and LIFT II[11]) or longitudinal posts, such as in this internship have insufficient data to be able to draw meaningful conclusions.

Implications for research and/or practice

The SPAI was effective at introducing new PAs into primary care and this test of concept internship can inform development of future similar schemes. The central employment model was valued by practices as it mitigated their risks, however, subsequent to the evaluation period it emerged that it was not feasible to continue with this as there were unexpected costs. The host trust classed the PAs interns as being on loan, not in a development post. Due to this classification, when invoicing practices and secondary care trusts to obtain the funding to pay the PA salary, the GP practices were classed as non-NHS organisations supplying staff resulting in value added tax implications. Thus, if a central funding model is to be successful, VAT implications and classifications of staff and organisations involved need to be clearly defined or risks may need to be underwritten to protect practices during this time. 
To support practices to deliver appropriate support for PA continuing professional development, nationally relevant, primary-care focussed guidance for is required. This should include the roles that new, UK PAs should be expected to undertake progressing through to what a more established PA in primary care could aspire to. Relevant governance information and agreed processes, where relevant, would help to reduce the uncertainty observed in this evaluation. Further, centralised training curricular, with adequate funding to deliver this, would benefit both new PAs and practices.

Within this predominantly split post SPAI model, after one year, most practices and PAs felt that further time was required to fully integrate the PA into their practice and many PA interns were still undertaking extended appointment times. Contributing factors include the broad range of presentations to primary care and the resultant time it takes to see the same presentation multiple times. Most evaluation participants felt that a two-year post-graduate programme in primary care would be appropriate to allow for a greater exposure to primary care and, possibly, a variety of secondary care specialties. The relative benefits and drawbacks of a rotational model, compared to a split longitudinal model would need to be further explored. It is not clear from this evaluation, nor the empirical literature, what the ideal internship length or design is. Time to reach a steady state in terms of appointment times, levels of support required from GPs and breadth of roles undertaken and population seen as well as impact of length of posts on supervisor engagement must be considered when planning future programmes and research on the same.

Development of trust between the PA intern and host practice was key, and an important consideration when operationalising policy initiatives such as PAs working across multiple practices within Primary Care Networks. The readiness for new PAs, or PAs reaching the end of the SPAI, for this type of working is unknown.

As practice familiarity with the role increases and practices become more aware of the different tasks PAs can undertake, the supporting role of the project manager, GP clinical lead and expert primary care PA for practices may reduce. However, at this current time these roles appear to be essential for supporting practices through uncertainties and difficulties in integrating new PAs into the primary care team.

\section{Conclusion}

The SPAI model was an effective way of integrating and implementing new PAs into UK primary care. High levels of support were required to achieve this successful integration, however, and PAs took time to develop their preference for, and autonomy within, primary care. To ensure successful ongoing integration of new PAs into UK primary care, it appears necessary to provide new PAs and their host practices time, informed support and financial resource and to provide PAs with a dedicated educational programme. Practices felt that for this model to be sustainable, recurrent funding, recognising the extent of adequate supervision with clear educational objectives aligned to a clear curriculum, need to be in place.

\section{Abbreviations}


FPA $=$ Faculty of Physician Associates

$\mathrm{GP}=$ General Practitioner

NSGPF = North Staffordshire GP Federation

PA = Physician Associate

PAMVR = Physician Associate Managed Voluntary Register

$\mathrm{PCN}=$ primary care network

RCP = Royal College of Physicians

SPAI = Staffordshire Physician Associate Internship

\section{Declarations}

\section{Ethical Approval}

Ethical approval was not required for this service evaluation. All people providing data were informed about the reasons for the evaluation and what would be happening to their data and all were free to choose whether they wanted to participate or not

\section{Availability of data and materials}

The datasets used and/or analysed during the current study are available from the corresponding author on reasonable request.

\section{Competing interests}

ST, EC, and JE were all GP partners at practices hosting a PA intern in the evaluated cohorts.

\section{Funding}

Funding for the Staffordshire Physician Associate Internship, and for employing the Physician Associate Interns, was provided by NHS England, Health Education England, the host employing Trust and individual host primary care practices. This evaluation was funded separately by Health Education England.

\section{Authors' contributions}

EC, JE, SL and MA designed the evaluation. ST, LM, ASJ and MA designed the internship and facilitated the evaluation to take place. EC undertook all data collection. EC and VS undertook primary data analysis. All authors have contributed to the writing of this paper. 


\section{Acknowledgements}

Thank you to the practice staff, physician associate interns and patients who were involved in providing data for this evaluation.

\section{References}

1. Physicians RC of. Physician Associate Managed Voluntary Register (PAMVR) [Internet]. Vol. 2019. Available from: https://www.fparcp.co.uk/employers/pamvr

2. NHS Digital. General Practice Workforce - 31 March 2020 [Internet]. Vol. 2020. 2020. Available from: https://digital.nhs.uk/data-and-information/publications/statistical/general-and-personal-medicalservices/final-31-march-2020

3. Ritsema T. Facult of Physician Associate Census results 2018 [Internet]. Vol. 2019. 2018. Available from: https://www.fparcp.co.uk/about-fpa/fpa-census

4. Digital NHS. General Practice Workforce 31st December 2019 [Internet]. Vol. 2020. 2020. Available from: https://digital.nhs.uk/data-and-information/publications/statistical/general-and-personalmedical-services/final-31-december-2019

5. NHS. NHS long term plan [Internet]. Vol. 2019. 2019. Available from: https://www.longtermplan.nhs.uk/

6. British Medical Association. Creating and running primary care networkds (PCNs) [Internet]. Vol. 2019. 2019. Available from: https://www.bma.org.uk/collective-voice/committees/generalpractitioners-committee/gpc-england/gp-contract-agreement-england/primary-care-networkspcns/creating-and-running-pcns\#Workforce

7. Howie N. Continuing professional development for Physician Associates in primary care. Educ Prim Care. 2017 Jul;28(4):197-200.

8. Lau R, Stevenson F, Ong BN, Dziedzic K, Treweek S, Eldridge S, et al. Achieving change in primary care--causes of the evidence to practice gap: systematic reviews of reviews. Implement Sci. 2016 Mar 22;11:40-4.

9. Drennan VM, Halter M, Brearley S, Carneiro W, Gabe J, Gage H, et al. Corrigendum: Investigating the contribution of physician assistants to primary care in England: a mixed-methods study. Heal Serv Deliv Res. 2016 May;2(16):137-8.

10. Physician associates in primary care | Health Education England [Internet]. [cited 2020 Aug 22]. Available from: https://www.hee.nhs.uk/our-work/primary-care/physician-associates-primary-care

11. LIFT | Health Education North West [Internet]. [cited 2020 Aug 21]. Available from: https://www.nwpgmd.nhs.uk/foundation/LIFT

\section{Figures}




\section{Five half-days a week in primary care}

\section{Four half-days in local hospital trust}

(e.g. acute medicine, emergency department, psychiatry or

$$
\text { acute surgery) }
$$

\section{Half-day \\ protected \\ learning}

\section{Repeated over a year}

Figure 1

Representation of how the week was divided up for SPAl interns over the single-year internship 